\title{
Topology and Design of Wide-Band 3D Metamaterials made of Periodically Loaded Transmission Line Arrays
}

\author{
Wolfgang J. R. Hoefer*, Poman P. M. So*, Dane Thompson** and Manos M. Tentzeris** \\ * Dept. of Electrical and Computer Engineering, University of Victoria, Victoria, BC, V8W 3P6 Canada \\ ** School of Electrical and Computer Engineering, Georgia Inst. Of Technology, Atlanta GA 30332-0250
}

\begin{abstract}
We propose a new topology for realizing artificial microwave materials that exhibit a negative refractive index over a wide frequency band. These metamaterials consist of a threedimensional transmission line mesh that is loaded with reactive series and shunt elements to create negative permittivity and permeability. The cubic-cell transmission line host network is a physical realization of the 3D distributed node TLM lattice which has the same low-frequency properties as the FDTD Yee scheme. This paper describes the topology, the theory, the design, and possible realizations of such a metamaterial. Its wave properties are validated by full-wave simulations using the time domain electromagnetic field simulation tool MEFiSTo-3D Pro.

Index Terms - Artificial materials, left-handed materials, metamaterials, negative refractive index materials, periodic structures, transmission line networks.
\end{abstract}

\section{INTRODUCTION}

Extensive research efforts are underway worldwide to create artificial materials that exhibit negative refractive index. One can classify existing approaches into two general categories, namely resonant and non-resonant periodic structures. Among the latter category belong the periodically loaded transmission line networks that have been demonstrated in 2002 by Iyer and Eleftheriades [1], [2], Caloz and Itoh [3], and Oliner [4]. They consist of host transmission line networks with embedded lumped series capacitors and shunt inductors. These periodic structures are capable of supporting backward waves as discussed by Ramo, Whinnery, and Van Duzer [5]. Analytical, numerical and experimental studies have confirmed that these loaded transmission line models exhibit indeed the peculiar properties predicted by Veselago [6].

Inspired by these realizations, So, Du and Hoefer [7] have created TLM-based 2D and 3D computational models of metamaterials, but so far, only one- and two-dimensional metamaterials have actually been realized in transmission line technology. In this paper we demonstrate for the first time how the transmission line approach can be extended to three space dimensions. Like the $1 \mathrm{D}$ and $2 \mathrm{D}$ cases, this 3D metamaterial is a realizable network of transmission lines with embedded series capacitances and shunt inductances. We present the theoretical concept, validate its wave properties through full-wave transient electromagnetic modeling, and develop a methodology for designing such a 3D metamaterial.

\section{THEORETICAL FOUNDATIONS}

The 3D distributed TLM node [8] is a realizable network model of Maxwell's equations. It has the same staggered (distributed) topology as the well-known FDTD scheme proposed by Yee. Its main advantage for the purpose of metamaterial design is that it can actually be built. Therefore, it is perfectly suitable as a host network for a transmissionline based metamaterial. All that is needed is the strategic embedding of reactive elements into this network. We will first summarize the wave properties of a 3D distributed node TLM network that consists of ideal transmission line sections, and determine the embedded reactive element values required to achieve a desired negative refractive index and wave impedance. We will then deal with the issues that arise when such networks are realized in a particular technology. Specifically, we must account for the effect of the shunt and series junctions that form the nodes of the real network, and the placement and implementation of the reactive embedded elements, and undesirable parasitic interactions between them.

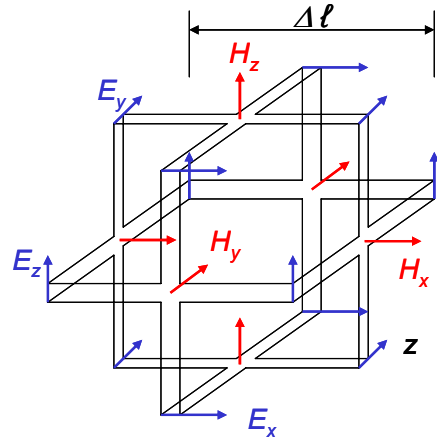

\section{Electric Field Component}

Magnetic Field Component

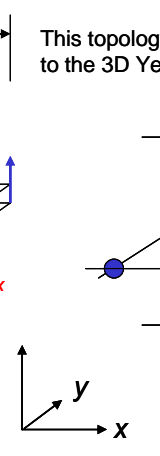
to the 3D Yee Cell in FDTD

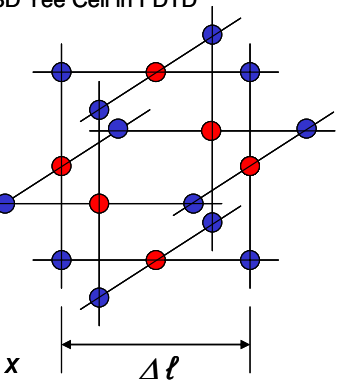

- Shunt Node

- Series Node
This topology is equivalent

Fig. 1. Unit cell of the 3D distributed node TLM network, consisting of staggered series and shunt connections of ideal transmission line sections. The field components are sampled at the same locations as in the FDTD Yee scheme.

Fig. 1 shows the unit cell or building block of the 3D distributed node TLM network. It consists of three shunt and three series nodes per cell - oriented in the three coordinate directions - that connect the lossless transmission line or link 
line sections of the mesh. It is, in fact, a transmission line equivalent of the FDTD Yee cell.

It has been well documented in the literature [9] that the low-frequency wave properties of this TLM network can be derived by using the axial equivalent cell, shown in Fig. 2 for $z$-polarized plane wave propagation in the y-direction. Due to symmetry the transverse link lines form reactive stubs that periodically load the main link line as shown below.

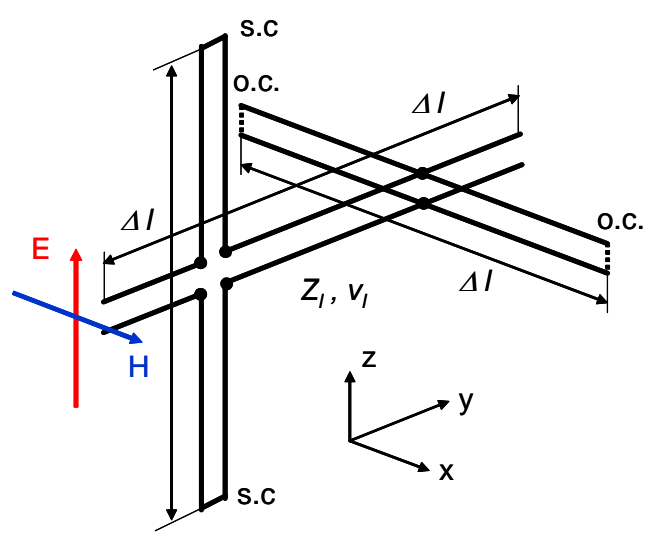

Fig. 2. Axial equivalent cell for z-polarized plane wave propagation in y-direction. Due to symmetry the transverse link lines are short-circuited at $\Delta \ell / 2$ from the series node and open-circuited at $\Delta \ell / 2$ from the shunt node.

\section{A. Wave Properties of the Host Network}

If the link lines have an inductance $L^{\prime}{ }_{\ell}$ per unit length and a capacitance $C_{\ell}^{\prime}$ per unit length, the characteristic link line impedance and phase velocity will be:

$$
Z_{\ell}=\sqrt{\frac{L_{\ell}^{\prime}}{C_{\ell}^{\prime}}} \text { and } \mathrm{v}_{\ell}=\frac{1}{\sqrt{L_{\ell}^{\prime} C_{\ell}^{\prime}}} .
$$

These expressions are isomorphic to the formulae for the wave impedance and phase velocity of a continuous medium with permeability $\mu_{\ell}=L_{\ell}^{\prime}$ and a permittivity $\varepsilon_{\ell}=C_{\ell}^{\prime}$. In the lowfrequency regime $(\Delta \ell / \lambda<<1)$ the short-circuited series stubs of the equivalent cell double the total series inductance, and the open-circuited shunt stubs double the total shunt capacitance per cell, thus forming a slow-wave structure with a network velocity $v_{n}$ that is half the link line velocity $v_{\ell}$. However, the network or Bloch impedance $Z_{n}$ will be the same as the link line impedance $Z_{\ell}$. We have

$$
\mathrm{v}_{n}=\frac{1}{\sqrt{2 L_{\ell}^{\prime} 2 C_{\ell}^{\prime}}}=0.5 \mathrm{v}_{\ell} \text { and } \mathrm{Z}_{n}=\sqrt{\frac{2 L_{\ell}^{\prime}}{2 C_{\ell}^{\prime}}}=Z_{\ell} .
$$

In the infinitesimal limit this periodic transmission line network models a continuous medium characterized by a permeability $\mu_{\mathrm{n}}$ and a permittivity $\varepsilon_{\mathrm{n}}$ that are related to the link line properties as follows: $\mu_{n}=2 L_{\ell}^{\prime} ; \varepsilon_{n}=2 C_{\ell}^{\prime} ; Z_{n}=\sqrt{\frac{\mu_{n}}{\varepsilon_{n}}}=Z_{\ell} ; v_{n}=\frac{1}{\sqrt{\mu_{n} \varepsilon_{n}}}=\frac{v_{\ell}}{2}$.

Its refractive index is also related to the link line properties:

$$
n_{n}=\frac{c}{v_{n}}=\frac{\sqrt{\mu_{n} \varepsilon_{n}}}{\sqrt{\mu_{0} \varepsilon_{0}}}=\sqrt{\frac{4 L_{\ell}^{\prime} C_{\ell}^{\prime}}{\mu_{0} \varepsilon_{0}}}=2 \frac{c}{v_{\ell}},
$$

where $c$ is the velocity of light in vacuo.

\section{B. Creation of a $3 D$ Network with Negative Refractive Index}

In order to make the refractive index of the axial equivalent network of Fig. 2 negative, we would ideally require transmission lines with negative series inductance and shunt capacitance. This is not physically possible, but we can make the total series impedance and shunt admittance of the cell negative, at least over a certain frequency range, by embedding a series capacitance and a shunt inductance in the cell, as shown in Fig. 3 and demonstrated in previous publications [1-5].

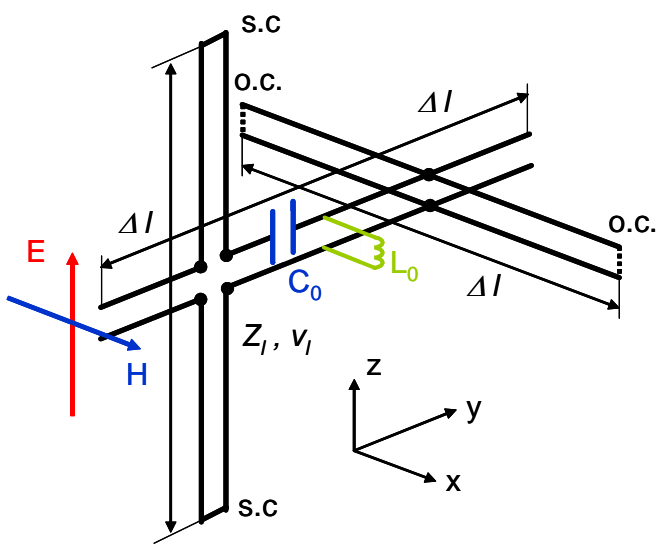

Fig. 3. Creation of an effective negative index of refraction by insertion of a series capacitance $\mathrm{C}_{0}$ and a shunt inductance $\mathrm{L}_{0}$ into the axial equivalent cell. The exact position of the elements is not critical and they could be integrated into the nodes as additional stubs.

To achieve a negative refractive index $n_{m}$ we must determine the values of $C_{0}$ and $L_{0}$ such that the total series impedance $Z_{s}$ and total shunt admittance $Y_{p}$ of the cell become both negative. These are given by

$$
\begin{aligned}
& Z_{s}=j 2 \omega L_{\ell}^{\prime} \Delta \ell+\frac{1}{j \omega C_{0}}=j \omega\left(2 L_{\ell}^{\prime} \Delta \ell-\frac{1}{\omega^{2} C_{0}}\right)=j \omega \mu_{m} \Delta \ell \\
& Y_{p}=j 2 \omega C_{\ell}^{\prime} \Delta \ell+\frac{1}{j \omega L_{0}}=j \omega\left(2 C_{\ell}^{\prime} \Delta \ell-\frac{1}{\omega^{2} L_{0}}\right)=j \omega \varepsilon_{m} \Delta \ell .
\end{aligned}
$$

Here we have introduced the effective permeability $\mu_{m}$ and permittivity $\varepsilon_{m}$ of the metamaterial that this model emulates. We can now determine the embedded elements $C_{0}$ and $L_{0}$ in terms of the desired metamaterial properties from (5) and (6) in which we replace the terms $2 L_{\ell}^{\prime}$ and $2 C^{\prime}{ }_{\ell}$ by the permeability $\mu_{n}$ and $\varepsilon_{n}$ of the host network given in (3): 


$$
\begin{aligned}
& C_{0}=-\frac{1}{\omega^{2} \Delta \ell\left(\mu_{m}-\mu_{n}\right)} . \\
& L_{0}=-\frac{1}{\omega^{2} \Delta \ell\left(\varepsilon_{m}-\varepsilon_{n}\right)} .
\end{aligned}
$$

The presence of the term $\omega^{2}$ in these expressions does not mean that $C_{0}$ and $L_{0}$ are frequency dispersive. $\omega$ is simply a design parameter that determines at which frequency a given $\mu_{m}$ and $\varepsilon_{m}$ are to be realized. However, once the embedded element values have been chosen, the resulting $\mu_{m}$ and $\varepsilon_{m}$ will be dispersive. For the low frequency approximation to be valid, the mesh parameter $\Delta \ell$ should be smaller than $1 / 10^{\text {th }}$ of the spatial wavelength in the metamaterial at the angular frequency $\omega$ and over the entire operating frequency range.

The final step towards the creation of a 3D metamaterial is to interconnect loaded axial equivalent cells into a full 3D distributed node network as demonstrated in Fig. 3.

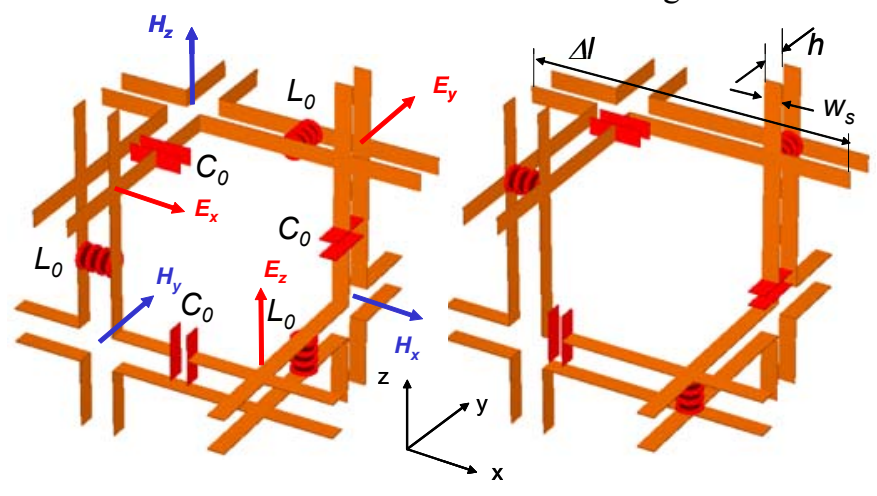

Fig. 4. Two possible topologies of transmission lines and embedded series capacitances $C_{0}$ and shunt inductances $L_{0}$ in conceptual distributed node realizations of a 3D metamaterial. The embedded elements can either be lumped or distributed depending on the technology used to build the structures. The substrate is not shown.

In accordance with the well-established theory of distributed node TLM networks, the 3D wave properties of the above structures approximate those of continuous negative refractive index materials in the low-frequency limit. A finite $\Delta \ell / \lambda$ ratio will lead to a dispersion error of the phase velocity commensurate with that of the host network or, for that matter, its equivalent Yee cell in FDTD.

\section{REALIZATION OF 3D METAMATERIALS}

The first step in the realization of such a $3 \mathrm{D}$ metamaterial is to select a type of transmission line that can easily be shuntand series-connected. One such type is the parallel strip line. Since it is essentially equivalent to a pair of identical back-toback microstrips with a common ground plane, we can make use of well-known empirical design expressions for microstrip. Furthermore, a first-order design that allows us to demonstrate and validate the negative refractive index properties of the 3D metamaterial concept, can be performed by replacing the parallel strips by their equivalent parallel plate waveguide model. This model is readily verified by $3 \mathrm{D}$ EM simulation.

\section{A. Parallel Strip Transmission Line Design on RT-Duroid}

We have designed a 3D host network using $1 / 4$ in. wide parallel strips on $1 / 16$ in. thick RT-Duroid with $\varepsilon_{\mathrm{r}}=2.2$. The dimensions and characteristics of the link lines obtained with empirical microstrip design formulae are given in Table I.

TABLE I

PROPERTIES OF PARALLEL STRIP LINK LINES

\begin{tabular}{|l|l|}
\hline Substrate thickness $\mathrm{h}$ & $1 / 16$ in. $(1.6 \mathrm{~mm})$ \\
\hline Substrate permittivity $\varepsilon_{\mathrm{r}}$ & 2.2 \\
\hline Strip width $\mathrm{w}_{\mathrm{S}}$ & $1 / 4 \mathrm{in} .(6.35 \mathrm{~mm})$ \\
\hline Effective dielectric constant $\varepsilon_{\mathrm{e}}$ & 1.9943 \\
\hline Characteristic impedance $\mathrm{Z}_{\ell}$ & $49.2 \Omega$ \\
\hline Equiv. parall. plate wguide width $\mathrm{w}$ & $8.61 \mathrm{~mm}$ \\
\hline Link line phase velocity $\mathrm{v}_{\ell}$ & $0.708 \mathrm{c}$ \\
\hline Inductance per unit length $\mathrm{L}_{\ell}{ }_{\ell}$ & $231.7 \mathrm{nH} / \mathrm{m}$ \\
\hline Capacitance per unit length $\mathrm{C}^{\prime}{ }_{\ell}$ & $95.77 \mathrm{pF} / \mathrm{m}$ \\
\hline Design frequency $\mathrm{f}$ & $230 \mathrm{MHz}$ \\
\hline Mesh parameter $\Delta \ell$ & $50 \mathrm{~mm}$ \\
\hline
\end{tabular}

The axial equivalent unit cell realized with these strips will not have exactly the properties predicted by the ideal model in Fig. 2 due to the finite cross-sectional dimensions of the parallel strips which introduce parasitics at the junctions. We have derived the following expressions for the total series inductance and shunt capacitance of the axial equivalent unit cell that account for them (dimensions are defined in Fig. 4):

$$
\begin{aligned}
& L_{t o t}=\mu_{n} \Delta \ell=L_{\ell}^{\prime}(2 \Delta \ell-h(2-p)-w(1-q)), \\
& C_{t o t}=\varepsilon_{n} \Delta \ell=C_{\ell}^{\prime}(2 \Delta \ell-w(2-s)-h(1-r)) .
\end{aligned}
$$

The coefficients $p, q, r$ and $s$ account for the parasitic effects of the junctions and can be determined by an electromagnetic simulation of the junction. They are slightly smaller than unity and depend on the strip width, height, and substrate permittivity; for very small values of $w$ and $h$, (9) and (10) tend towards the ideal case represented by (3). For our design we have obtained the following values for the junction coefficients: $p=s=0.99$ and $q=r=0.73$. The resulting host network wave properties are presented in Table II.

We can now calculate the values of $C_{0}$ and $L_{0}$ required for creating a metamaterial with a desired negative refractive index. If we choose $\mu_{m}=-\mu_{n}$ and $\varepsilon_{m}=-\varepsilon_{n}$, we obtain a refractive index $n_{m}=-n_{n}$ and a Bloch impedance $Z_{m}=Z_{n}$. (7) and (8) yield the required embedded reactances at the design frequency $(f=230 \mathrm{MHz})$ at which the guided wavelength in the material is about $10 \Delta \ell$. At this frequency we expect a dispersion error (discretization error) of about one percent. 


\section{TABLE II}

Properties of Parallel Strip Line Host Network

\begin{tabular}{|l|l|}
\hline Total series inductance per cell $\mathrm{L}_{\text {tot }}$ & $22.26 \mathrm{nH}$ \\
\hline Total shunt capacitance per cell $\mathrm{C}_{\text {tot }}$ & $8.703 \mathrm{pF}$ \\
\hline Host network permeability $\mu_{\mathrm{n}}=\mathrm{L}_{\text {tot }} / \Delta \ell$ & $445.19 \mathrm{nH} / \mathrm{m}$ \\
\hline Host network permittivity $\varepsilon_{\mathrm{n}}=\mathrm{C}_{\text {tot }} / \Delta \ell$ & $174.06 \mathrm{pF} / \mathrm{m}$ \\
\hline Host network wave impedance $\mathrm{Z}_{\mathrm{n}}$ & $50.57 \Omega$ \\
\hline Host network refractive index $\mathrm{n}_{\mathrm{n}}$ & 2.639 \\
\hline Host network phase velocity $\mathrm{v}_{\mathrm{n}}$ & $0.535 \mathrm{v}_{\ell}=0.3789 \mathrm{c}$ \\
\hline$\Delta \ell / \lambda_{\mathrm{n}}$ at $230 \mathrm{MHz}$ & 0.101 \\
\hline Mesh parameter $\Delta \ell$ & $50 \mathrm{~mm}$ \\
\hline
\end{tabular}

We obtain $L_{0}=27.51 \mathrm{nH}$ and $C_{0}=10.76 \mathrm{pF}$ that are required to create a refractive index $n_{m}=-2.639$ and a wave impedance $Z_{m}=50.57 \Omega$ at $230 \mathrm{MHz}$.

\section{B. Validation of the Design Example}

We have computed the wave properties of the host network before and after embedding the reactive elements, using the full-wave time domain EM simulator MEFiSTo-3D Pro.

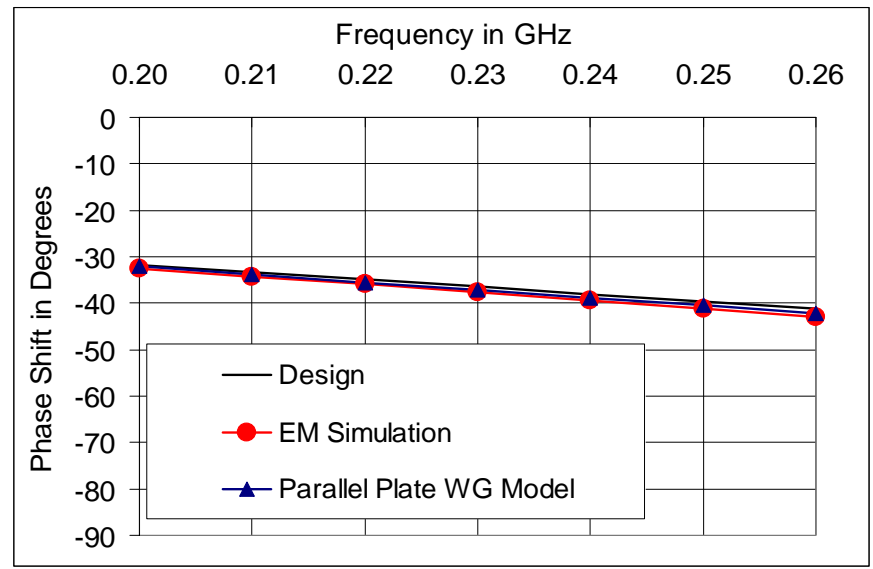

Fig. 5. Phase of $S_{21}$ across a single cell of the parallel strip host network. The negative phase shift indicates positive phase velocity.

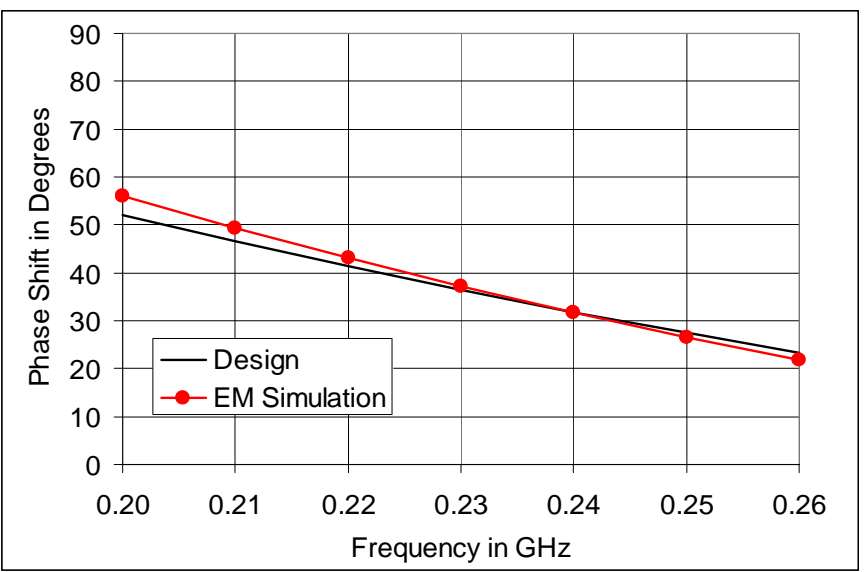

Fig. 6. Phase of $S_{21}$ across a single cell of the metamaterial. The positive phase shift indicates negative phase velocity. The slight curvature indicates that the metamaterial is only slightly dispersive.
In particular, have computed the angle of $S_{21}$ of one axial equivalent cell to verify the magnitude and sign of the network phase velocities $v_{n}$ and $v_{m}$ from 200 to $260 \mathrm{MHz}$. Fig. 5 compares the predicted and simulated phase shift across a single cell of the host network without the embedded elements. Fig. 6 shows the same for the metamaterial created by embedding the designed elements $L_{0}$ and $C_{0}$ into this host network. In the EM simulation, $L_{0}$ and $C_{0}$ were modeled by a short-circuited shunt stub and an open-circuited series stub, respectively. Note that the phase angle is $-38^{\circ}$ in the host cell and $+38^{\circ}$ in the metamaterial cell at the design frequency of $0.23 \mathrm{GHz}$. The validity of the concept has thus been convincingly demonstrated by full-wave EM simulation, opening the way to the production of an experimental 3D metamaterial prototype based on a parallel strip transmission line network.

\section{CONCLUSION}

A new methodology for creating 3D metamaterials based on periodically loaded transmission line networks has been proposed and validated by a design example and by 3D fullwave electromagnetic simulations. The design approach will be used to realize an experimental prototype of a 3D metamaterial based on parallel strip transmission lines.

\section{REFERENCES}

[1] A. K. Iyer and G. V. Eleftheriades, "Negative refractive index metamaterials supporting 2-D waves," IEEE MTT S Int. Microwave Symp. Dig., vol. 2, pp. 1067-1070, June 2002.

[2] G. V. Eleftheriades, A. K. Iyer, and P. C. Kremer, "Planar Negative Refractive Index Media Using Periodically L-C Loaded Transmission Lines", IEEE Trans. Microwave Theory Tech., vol. 50, no. 12, pp. 2702-2712, December 2002.

[3] C. Caloz and T. Itoh, "Application of the transmission line theory of lefthanded (LH) materials to the realization of a microstrip LH transmission line," IEEE AP-S Int. Symp. Dig., vol. 2, pp. 412-415, June 2002.

[4] A. A. Oliner, "A periodic-structure negative-refractive-index medium without resonant elements," USNC/URSI Nat. Radio Science Meeting, San Antonio, TX, p. 41, June 2002.

[5] S. Ramo, J. R. Whinnery, and T. Van Duzer, Fields and Waves in Communication Electronics, 3rd Edition., pp. 263-264, New York: Wiley, 1994.

[6] V. G. Veselago, "The electrodynamics of substances with simultaneously negative values of $\varepsilon$ and $\mu$ ", Sov. Phys.-Usp., vol. 10, no. 4, pp. 509-514, January-February 1968.

[7] P. P. M. So, H. Du and W. J. R. Hoefer, "Modeling of Metamaterials with Negative Refractive Index Using 2D-Shunt and 3DSCN TLM Networks", IEEE Trans. Microwave Theory Tech., vol. 53, no. 5, April 2005. (In press)

[8] S. Akhtarzad, and P. B. Johns, "Solution of Maxwell's equations in three space dimensions and time by the TLM method of numerical analysis," Proc. Inst.Elec. Eng. Vol. 122, no. 12, pp. 1344-1348, December 1975.

[9] W.J.R. Hoefer, "The Transmission Line Matrix (TLM) Method", Chapter 8 in "Numerical Techniques for Microwave and Millimeter Wave Passive Structures", edited by T. Itoh, New York, John Wiley \& Sons, pp. 496-591, 1989. 